\title{
Etat d'avancement du programme Eurêka EU205 "laser à excimères de fortes puissances moyennes pour usage industriel"
}

\section{Stehle}

Societé SOPRA, 26 rue P. Joigneaux, 92270 Bois-Colombes, France

Les lasers à excimères sont apparus au début des années 80 . Pendant dix ans environ, ils ont été presque uniquement utilisés en intrumentation scientifique.

Au fur et à mesure des besoins, leur puissance mojenne a évolué à partir de quelques Watts pour atteindre ensuite $100 \mathrm{~W}$ vers 1990.

Le Programme Eurêka, lancé en 1987, a pour vocation de développer des lasers à excimères de forte puissance moyenne : $1 \mathrm{KW}$, pour usage industriel (XeCl, $\lambda=308 \mathrm{~mm})$. constituees.

Plusieurs équipes de recherche chez différents partenaires industriels européens ont été

Nous développerons plus particulièrement dans cette présentation les voies retenues par SOPRA coordonnateur français du projet EU205 - et Laserdot - filiale de la holding Unilaser, du groupe Aérospatiale -

Le Programme Burêka EU205 est structuré autour des industriels et organismes suivants, répartis par nationalités :

\begin{tabular}{|llllll|}
\hline FRANCE & ALLEMAGNE & ROYAUME-UNI & GRECE & PAYS-BAS & SUEDE \\
\hline $\begin{array}{l}\text { SOPRA } \\
\text { Laserdot }\end{array}$ & $\begin{array}{l}\text { Rofin Sinar (Siemens) } \\
\text { Lambda Physik }\end{array}$ & $\begin{array}{l}\text { Oxford Lasers } \\
\text { Exitech }\end{array}$ & FORTH & FOM & Permanova \\
\hline
\end{tabular}

En France, les deux industriels bénéficient du soutien de l'ONERA pour SOPRA et du CNRS pour Laserdot et SOPRA.

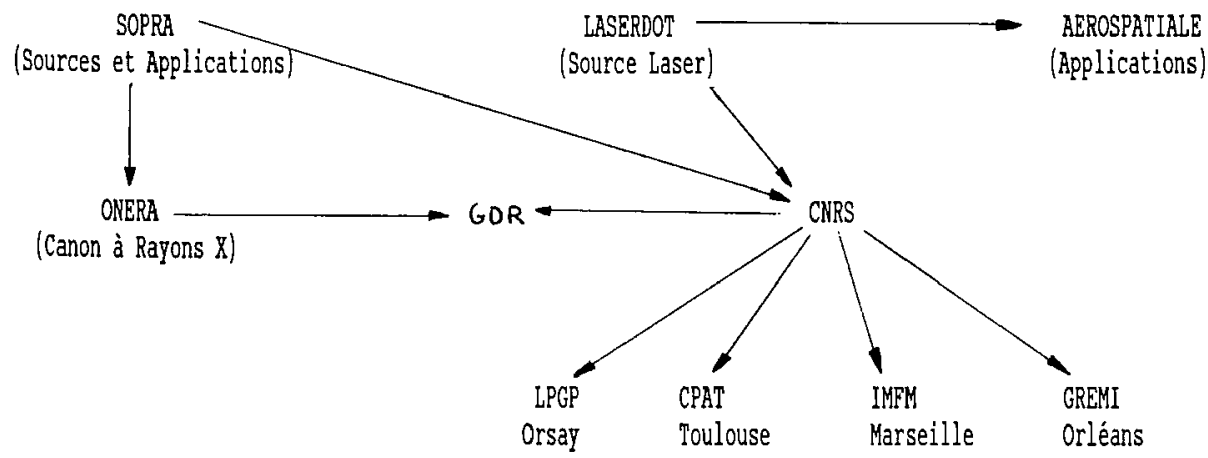


Depuis le début du projet, SOPRA a choisi de développer une source laser d'un Kilowatt, orientée vers les hautes énergies par tir : plus de 10J. L'énergie laser, extraite par unité de volume de wilieu gazeux amplificateur, étant limitée par la saturation du gain, une grande énergie par tir implique de pouvoir exciter un grand volume (5 litres).

Plusieurs tppes de préionisation ont été explorés :

$$
\begin{aligned}
& \text { I Arcs UV } \\
& \text { I Corona, décharges rampantes, } \\
& \text { I Rayons \& (de 50keV jusqu'à 150ReV), }
\end{aligned}
$$

Pour les grands volumes de gaz, la préionisation par rapons 8 offre les meilleurs résultats.

SOPRA bénéficie de l'appui du Laboratoire d'Optique Quantique de l'ONERA à Palaiseau.

En effet, l'équipe de M. D. Pigache apait développé un canon à électrons secondaires qui avait déjà été testé avec succès sur le laser L.U.X. de l'IMFM avant le début du Programme Eu205. Dans le cadre du projet, plusieurs canons de tailles supérieures et plus performants en terme de dose et d'uniformité de rayons $X$ ont été construits et testés avec succès.

Ceci a permis à SOPRA de développer une technologie de laser de fortes énergies par tir.

\begin{tabular}{|c|c|c|c|}
\hline DATE & MAQUETTE & PERFORMANCES ATTEINTES & PUISSANCE MOYENNE \\
\hline 1987 & Proto 1 & $0.6 \mathrm{~J} / \mathrm{tir}$ & \\
\hline 1988 & Proto 2 & $2 \mathrm{~J} / \mathrm{tir}$ & \\
\hline 1989 & DENEB & $5 j /$ tir & \\
\hline $02 / 1990$ & DENEB & $4 \mathrm{~J} / \mathrm{tir}$ à $50 \mathrm{~Hz}$ & $200 \mathrm{w}$ \\
\hline $11 / 1991$ & VECA & $10 \mathrm{~J} / \mathrm{tir}$ & \\
\hline $01 / 1992$ & VEGA & 15J/tir & \\
\hline $08 / 1992$ & VEL & $13 \mathrm{~J} /$ tir à $\mathrm{BOHz}$ & $1 \mathrm{KW}$ \\
\hline
\end{tabular}
L'optimisation paramétrique sur différentes maquettes intermédiaires a aussi permis de franchir rapidement plusieurs étapes. Les principales sont reportées dans le tableau suivant:

Les meilleures performances atteintes sont:

I Puissance moyerne maximale :
$\mathrm{Pa}=1 \mathrm{KW}$
selon deus modes :
$13 \mathrm{~J} /$ tir à $\mathrm{f}=80 \mathrm{~Hz}$
ou $\quad 10 \mathrm{~J} /$ tir à $\mathrm{f}=100 \mathrm{~Hz}$

1 Energie par tir maximale :

E : $15 \mathrm{~J}$ avec un rendenent de $2 \%$

I Meilleur rendement :

$\rho=2.7 \%$, quand $E=10 \mathrm{~J}$

Le laser VEL (1KW) est la première source mondiale $\mathrm{WV}(\lambda=308 \mathrm{~nm}$, XeCl) a avoir atteint le kilowatt et place SOPRA en position de leader devant les concurrents japonais, européens et américains. 


\section{Les applications}

Compte tenu de l'originalité de la démarche poursuivie, plusieurs grandes lignes d'application se détachent, surtout celles motivées par le concept de grande énergie par impulsion, et celles pour lesquelles le recourrement (balayage) de faisceau est proscrit.

Exemples :

I recuit de Siliciun (écrans plats - "TFT-FPD"),

I aarquage de grandes dimensions (verres, céramiques),

1 polywérisation,

I ablation de polymères,

I décapage de peintures,

I décontamination nucléaire,

1 modification de rugosité (tribologie),

1 suppression de criques (résistance à la fatigue),

I source de rayons $\mathrm{X}$,

I amplification sub-picoseconde.

etc ...

Le laser à excimère de grande puissance moyenne $(0.5 \mathrm{WW}$, XeCl, $\lambda=308 \mathrm{~nm})$ développé par Laserdot, dans le cadre du projet Eurêka EU205, est aussi caractérisé par la mise en oeuvre de concepts de physique innovants par rappport aux différents concurrents japonais et européens.

Le principe retenu par Laserdot est celui du laser à excinère photo-déclenché inventé par $\mathrm{M}$. Bernard Lacour. Jusqu'à présent, le photo-déclenchement est assuré par une préionisation créée par effet Corona.

Compte tenu du concept choisi, le projet de la société Laserdot conduit à un prototype de 500w selon $0.8 \mathrm{~J} /$ tir à une fréquence de répétition de $650 \mathrm{~Hz}$. Des fréquences plus élevées $(750 \mathrm{~Hz})$ et des énergies par tir légèrement plus élevées ont été atteintes séparément, mais le meilleur compromis est atteint avec $0.8 \mathrm{~J}$ \& $650 \mathrm{~Hz}$, soit 500W (Eté 1992).

Le laser photo-déclenché se singularise des autres projets conduits à l'étranger sur le même créneau (Lambda Physik - 1J × 500Hz -, British herospace - 1J \& 1KHz -, NCLR - 1J \& $1 \mathrm{KHz}$-, ENEA - IJ \& $1 \mathrm{KH} 2$-, Mitsubichi, Toshiba, NEC, etc ...) par le fait que la commutation principale de l'énergie stockée dans des condensateurs, est assurée par le "photo-déelenchement-Corona" lui-même et non par des thyratrons comme traditionnellement; ceci constitue un grand avantage (coût, fiabilité).

Comme on l'a vu précédenment, les deux industriels français ont une démarche complémentaire et non-concurrentielle.

En plus de la relation qui lie l'ONERR à SOPRA, à travers le développement du canon de préionisation à rayons $X$, plusieurs laboratoires de recherche du CNRS (IMEM, LPGP, CPAT, GREMI) associant sis équipes différentes sont regroupés au sein d'un GDR (Groupement De Recherche). 
L'apport du GDR-CNRS n'est pas des moindres et contribue au succès de ce projet EU205.

En effet, dans le cadre de ce GDR, les études de modélisation des différentes maquettes, tant sur le plan électrique que des phénomènes élémentaires, sont abordées. La cinétique du milieu laser I est traitée abondamment avec des nodèles à zéro dimension qui produisent une correspondance acceptable entre les expériences et les calculs résultant des modèles.

Plusieurs thèses ont été soutenues sur ces thèmes de recherche.

De plus, l'IMPM mène aussi des expérimentations en vraies grandeurs jusquu'à des fréquences de l'ordre du kilohertz et aborde ainsi les problèmes de propagation d'ondes de chocs dans la cavité laser et les poyens d'y remédier (amortisseurs, absorbants).

Conclusion

Ainsi, en conclusion, on peut affirmer que ce progranme industriel Euréka Eu205 est un succès au niveau des résultats déjà acquis ( $1 \mathrm{WW}$ chez SOPRA et $0.5 \mathrm{WW}$ chez Laserdot). La contribution des autres organismes associés à ce projet (ONERA et CNRS) se déroule de façon exemplaire.

Il est à souhaiter que l'évolution des structures mises en place par le CNRS (GDR/Excimère) suive l'évolution future du projet EU205 qui ira prochainement de la recherche actuelle vers le développement des sources lasers et de leurs applications. 